\title{
Pegylated liposomal doxorubicin in malignant pleural mesothelioma: a possible guardian for long-term survival
}

This article was published in the following Dove Press journal:

OncoTargets and Therapy

26 September 2012

Number of times this article has been viewed

\author{
Paul Zarogoulidis ${ }^{1,2}$ \\ Maria Mavroudi' \\ Konstantinos Porpodis' \\ Kalliopi Domvri \\ Antonios Sakkas ${ }^{3}$ \\ Nikolaos Machairiotis' \\ Aikaterini Stylianaki' \\ Anastasios Tsiotsios' \\ Nikolaos Courcoutsakis ${ }^{4}$ \\ Konstantinos Zarogoulidis' \\ 'Pulmonary Department-Oncology \\ Unit, "G Papanikolaou” General \\ Hospital, Aristotle University \\ of Thessaloniki, Thessaloniki, \\ Greece; ${ }^{2}$ Pulmonary Department- \\ Interventional Unit, Ruhrland Klinik, \\ University of Essen, Essen, Germany; \\ ${ }^{3}$ Department of Pathology, \\ "G Papanikolaou" General Hospital, \\ Aristotle University of Thessaloniki, \\ Thessaloniki, Greece; ${ }^{4}$ Department \\ of Radiology, University General \\ Hospital of Alexandroupolis, \\ Democritus University of Thrace, \\ Alexandroupolis, Greece
}

Correspondence: Paul Zarogoulidis "G Papanikolaou" General Hospital, Aristotle University of Thessaloniki, Exohi 570 10, Thessaloniki, Greece

Tel +30 6977271974

Fax +302310 992433

Email pzarog@hotmail.com
Abstract: Malignant pleural mesothelioma is a rare and aggressive malignancy of the pleura correlated with exposure to asbestos, with a medium survival of 11-12 months after diagnosis. A case of a 67-year-old male who had previously worked in the asbestos industry and is a current smoker is reported. The computed tomography evaluation revealed a right pleural mass with pleural thickening, and the pleural biopsy confirmed a diagnosis of malignant pleural mesothelioma. He was treated with chemotherapy consisting of etoposide, paclitaxel, and pegylated liposomal doxorubicin hydrochloride. After completion of chemotherapy, radiologic evaluation confirmed a reduction of pleural thickening and improvement in his symptoms. A complete presentation of each drug formulation and characteristics are also included in this paper. The patient's follow-up is continuing, and computed tomography reveals stable disease 9 years after initial examination.

Keywords: mesothelioma, asbestos, pegylated liposomal doxorubicin

\section{Introduction}

Malignant pleural mesothelioma (MPM) is a rare and highly aggressive tumor that derives from pluripotential mesothelial cells. ${ }^{1}$ Histologically, it is divided into three subtypes: epithelioid, which is the most common type, sarcomatoid, and mixed type. The main risk factor of MPM is the exposure to asbestos, a group of crystallinehydrated silicate minerals that, due to its mineral properties such as thermal and cold resistance, tensile strength, and low cost, are used in a variety of manufacturing goods such as tiles, ceilings, cement products, and fabrics. Asbestos is divided into two groups: the serpentine form composed of spiral fibers (chrysotile), which is suitable for making fabrics, and the amphibole form composed of short and linear fibers (anthophyllite, crocidolite, tremolite, actinolite, and amosite), which is most suitable for the construction of tiles and pipes. It is believed that the amphibole form is more likely to cause chronic irritation that can lead to malignant mesothelioma. To a lesser degree, other risk factors include the exposure to other mineral fibers such as erionite, simian virus 40 , and radiation. This type of neoplasm is not correlated with smoking, in contrast to the majority of other types of lung cancers. ${ }^{2,3}$

MPM demonstrates increasing incidence worldwide especially in industrialized countries, due to previous exposure to asbestos. ${ }^{4}$ The prognosis of these patients is poor, with a median survival of 11-12 months and only a small proportion of patients surviving 2 years after diagnosis. Differences in survival are associated with environmentally correlated factors such as the duration and quality of asbestos exposure, and are also 
correlated with age at the time of diagnosis, gender, health status, and histological subtype of the tumor. Poor performance status, advanced age, male sex, and sarcomatoid subtype are all poor prognostic factors. ${ }^{5,6}$ The disease usually manifests with thoracic pain and dyspnea, and the chest X-ray shows pleural effusion - a widespread pleural thickening and reduction in volume of the hemithorax. Thoracoscopy or open pleural biopsy is necessary for diagnosis. ${ }^{7}$ The immunochemistry of the biopsy specimen is also very important and can help determine the differential diagnosis between MPM and metastatic lung adenocarcinomas. Markers such as keratin-5, calretinin, podoplanin, and Wilms tumor gene-1 are sensitive for MPM - particularly for the epithelioid and mixed subtypes. In contrast, polyclonal carcinoembryonic antigen, thyroid transcription factor-1, and monoclonal antibody-31 (MOC-31) are markers sensitive for adenocarcinoma but negative for MPM. ${ }^{8}$ The therapeutic options in patients with MPM usually include surgery, chemotherapy, and adjuvant radiotherapy. In selected patients, multimodality treatment with surgical resection followed by radiation and systemic chemotherapy seems to have benefits, but the standard therapy for advancedstage patients is still pemetrexed and cisplatin as first-line chemotherapy. However, several other chemotherapy regimens have been used as the first-line treatment alone or in combination, with positive results, indicating possible alternatives. ${ }^{9-13}$

In the current case presentation, Caelyx ${ }^{\circledR}$ (pegyliertes liposomales doxorubicin; Janssen Pharmaceuticals, Inc., Titusville, NJ) $20 \mathrm{mg}$ was used as the first-line treatment in combination with paclitaxel $\left(175 \mathrm{mg} / \mathrm{m}^{2}\right)$ and etoposide $\left(200 \mathrm{mg} / \mathrm{m}^{2}\right.$ on days one to three), which presented a favorable outcome as a first-line treatment. The characteristics of the three drugs are presented along with the characteristics of the malignant mesothelioma disease. Most recent studies have focused on demonstrating better genetic and molecular events that occur during asbestos exposure with the hope of leading to a new class of anticancer agents. ${ }^{2,4}$ This paper presents a long-term survivor through the combination of an anthracycline agent, taxane, etoposide, and cyclophosphamide; this combination treatment has previously demonstrated prolonged survival. ${ }^{14}$

\section{Case presentation}

A 67-year-old male pensioner attended the emergency department complaining of chest pain, especially in the right hemithorax, and dyspnea. The symptoms first presented 1 month prior to initial examination. The patient did not mention any cough, hemoptysis, fever, or weight loss. His medical history did not include any comorbidities and he was not taking any medication. The social history demonstrated that the patient was an ex-worker in the asbestos industry and a current smoker (30 cigarettes per day for 47 years). Lung auscultation showed that there was a reduction of respiratory sound in the right lung. The X-ray showed thickening of the right pleura and the patient was sent for a computed tomography (CT) scan. The contrast-enhanced chest CT scan showed a right peripheral pleural mass with pleural thickening and areas with braces, especially in the medium and lower right lobe, with small pretracheal lymph nodes (Figure 1). Round atelectasis subpleural focuses were revealed in the right medium and lower lobe. An abdomen CT showed a hypodense mass in the right adrenal gland $(5 \times 3 \mathrm{~cm})$. At this point the possibility of MPM (epithelial) with metastasis in the left adrenal gland was considered. To confirm the diagnosis, thoracoscopy was performed to obtain a pleural biopsy, with a positive result for MPM. A bone scan was also performed and was negative for disease.

The patient was treated in the authors' department with eight cycles of paclitaxel $\left(175 \mathrm{mg} / \mathrm{m}^{2}\right)$, pegylated liposomal doxorubicin hydrochloride (Caelyx $20 \mathrm{mg} / \mathrm{m}^{2}$ ), and etoposide $\left(200 \mathrm{mg} / \mathrm{m}^{2}\right)$, and he continued with three cycles of maintenance therapy with etoposide $\left(100 \mathrm{mg} / \mathrm{m}^{2}\right.$ on days one to five) and three cycles of cyclophosphamide $\left(150 \mathrm{mg} / \mathrm{m}^{2}\right.$ on days one to seven). The patient refused to complete the maintenance therapy and so he entered the follow-up period. The chest CT scans showed a reduction of the pleural thickening, and no pathological lymph nodes were observed (Figure 2). An additional biopsy was performed 5 years after the initial examination and the immunohistochemistry was positive for the following staining profile: Anti-pan Cytokeratin antibody (AE1/AE3), Wilms tumor gene-1, calretinin, D2-40, negative polyclonal carcinoembryonic

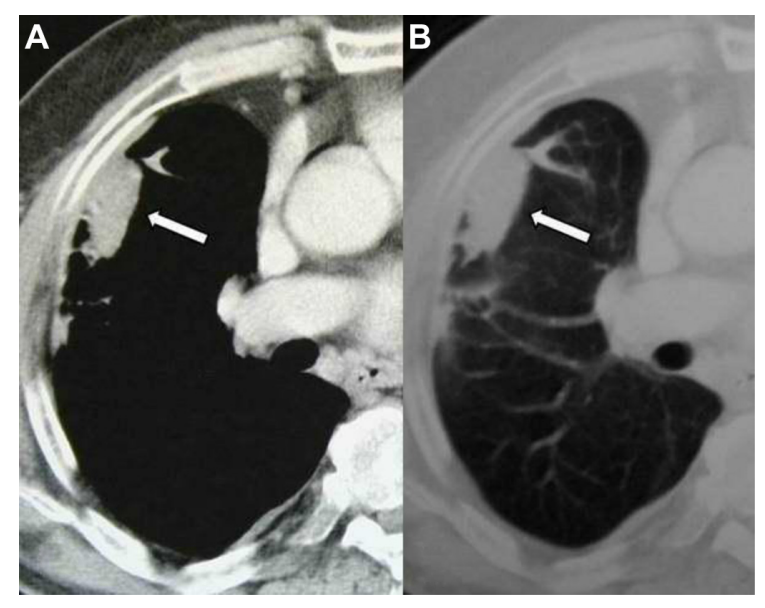

Figure I Axial computed tomography images of the right lung in the (A) mediastinal and (B) pulmonary window demonstrate the pleural mass corresponding to mesothelioma (initial examination). 


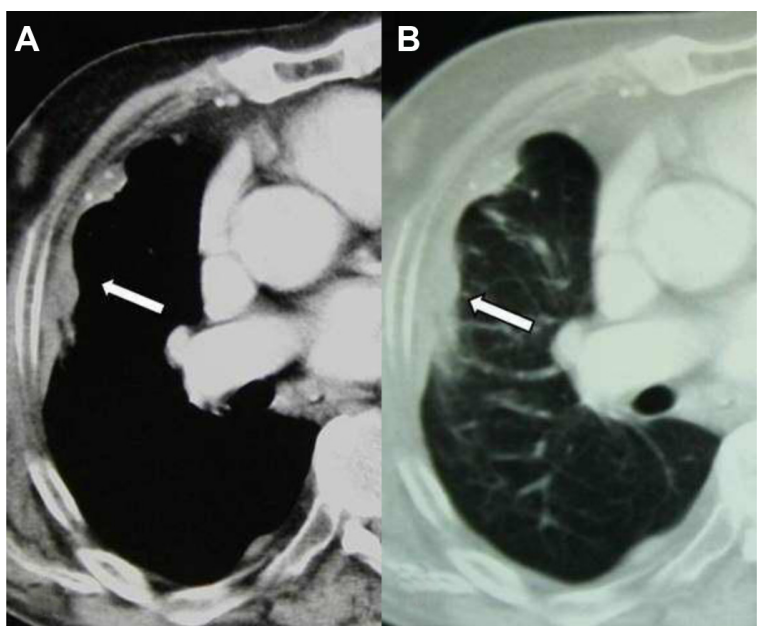

Figure 2 Axial computed tomography images of the right lung in the (A) mediastinal and (B) pulmonary window 9 years after initial examination show that the bulky side of the mesothelioma has slightly decreased.

antigen, and thyroid transcription factor-1. Metastasis in the adrenal gland remained stable. Bone scans and brain CT scans were not pathological. No confirmation was made on the malignancy of the adrenal gland. Pleural thickness at diagnosis was $90 \mathrm{~mm} \times 20 \mathrm{~mm}$, and $40 \mathrm{~mm} \times 15 \mathrm{~mm}$ at the end of the first-line treatment. Follow-up has continued for 9 years; the disease is stable without any disease progression.

\section{Discussion}

MPM is an uncommon but aggressive tumor of the pleura that is strongly correlated with chronic exposure to asbestos, with prevalence in males. MPM usually invades locally; however, metastases can be observed in the contralateral lung, bones, liver, or peritoneum. ${ }^{15}$ Patients usually manifest chest pain, dyspnea, and pleural effusion. Imaging examination is very important for diagnosis and during the follow-up period. To confirm the diagnosis, it is necessary to obtain a pleural biopsy with thoracoscopy and to examine the biopsy with immunohistochemistry. There are six types of asbestos fibrous silicates (actinolite, grunerite, anthophyllite, chrysotile, crocidolite, and tremolite). All types of asbestos fibers are capable of causing mesothelioma; however, only three types have been used commercially (white asbestos [chrysotile], brown asbestos [amosite], and blue asbestos [crocidolite]).

MPM incidence has been reported to be increasing in developing industrialized countries; new data indicate an escalation in Brazil, Thailand, and Egypt. In Greece, there is a $2 \%$ occupational morbidity attributed to pleural mesothelioma. The disease is still underreported by the national health agencies. ${ }^{16}$ Until 1995, Greece was one of the world's top seven suppliers of asbestos (chrysotile). More than 150,000 tons were produced and processed at various asbestos cement factories. Several products such as asbestos-containing brakes and fireproofing materials were also produced in Greece. Blue asbestos usage was banned by law in Greece (article 1154/93) on December 31, 2004; Greece became the last of the 15 European Union member states to ban the use of all forms of asbestos as per the European Union directive. ${ }^{16,17}$

The patient in the current report was treated with etoposide, paclitaxel, and pegylated liposomal doxorubicin as first-line treatment. This type of doxorubicin is coated with methoxy polyethylene glycol that decreases the interaction of the lipid membrane with the plasma components and with the reticuloendothelial system. The liposomal encapsulation and "stealth" ability has added additional advantages to the drug formulation. This drug formulation increases the half-life of doxorubicin and also the liposomal deposition in the tumor tissue by 100 -fold, so drug toxicity is reduced, especially cardiac toxicity and palmar-plantar erythrodysesthesia. Previously published phase I and II studies have shown good tolerance in patients with a dose of $45 \mathrm{mg} / \mathrm{m}^{2}$ Caelyx every 28 days. ${ }^{18,19}$ A previous study using first-generation anthracyclines presented adverse effects, restricting their usage due to cardiotoxicity and general intolerance. ${ }^{20}$ The polyethylene glycol coating (dual layer) augmented the efficacy of the drug formulation by adding a sustained-release effect. With this mode of drug release, the concentration of the formulation did not peak instantly after administration and therefore reduced the side effects; however, acute hypersensitivity reaction to the compound has been observed. ${ }^{21}$ Polymers have the ability to inhibit opsonization of the liposomes by plasma proteins and therefore increase the half-life of liposomal drugs. ${ }^{22-24}$ Prolonged systemic circulation enhances micrometastasis control. In addition, polyethylene glycol reduces endothelial cell interaction, and in turn the rate of extravasation is reduced. ${ }^{25}$ Pegylated liposomal doxorubicin is extensively used for the treatment of cancer, especially breast cancer, ovarian cancer, and Kaposi's sarcoma, and has shown to be an active drug for MPM treatment in phase II studies..$^{9-11,21}$ Pegylated liposomal doxorubicin should not be disregarded, and should be used as an alternative in patients who cannot tolerate other first-line chemotherapy regimens.

On the other hand, taxanes (paclitaxel, docetaxel) are a group of chemotherapy drugs with antiproliferative action. In particular, they arrest cells in the G2/M phase of the cell 
cycle, inhibit microtubule depolymerization, and reduce tumor angiogenesis. In addition, it is possible that paclitaxel is able to induce the gene expression of tumor necrosis factor- $\alpha$, and thus cell death. Taxanes are used with good results in the treatment of locally advanced nonsmall cell lung cancer, in combination with cisplatin or radiotherapy. ${ }^{26}$ In MPM, taxanes in combination with cisplatin also seem to have good results in inhibiting cell proliferation. ${ }^{27,28}$ The patient in the current report showed improvement in his symptoms and, after the completion of chemotherapy, a reduction of pleural thickening and elimination of the pathological lymph nodes.

Etoposide(VP1 6-2 13) is a semisynthetic podophyllotoxin derivative and an inhibitor of DNA topoisomerase II. It has the ability to stabilize DNA strand breaks. ${ }^{29}$ Etoposide has presented favorable results in a variety of malignancies. ${ }^{30}$ The drug is schedule dependent and acts on the late S and early G2 phases of the cell cycle. ${ }^{31}$ This compound has demonstrated efficiency in small cell lung cancer and is administered orally or by intravenous infusion. ${ }^{32-34}$ Modest leukopenia was observed in the oral route of administration. ${ }^{35}$ In mesothelioma, there are scarce data available of the efficacy of etoposide, nevertheless the European Organization for Research and Treatment of Cancer Lung Cancer Cooperative Group initiative presented efficacy data (intravenous and oral) with two phase II trials. ${ }^{35,36}$ Data presented in these trials and in another study are in favor of etoposide administered orally for MPM within a combination..$^{35-37}$

Cyclophosphamide is a nitrogen mustard alkylating agent. It is used in a variety of diseases including cancer and autoimmune disorders. It is converted in the liver from the prodrug to the active chemotherapeutic agent. The cytotoxicity of cyclophosphamide is dose dependent. ${ }^{38}$ Cyclophosphamide has presented efficacy in combination with cisplatin and doxorubicin in MPM. ${ }^{14,39}$ Cyclophosphamide has the additional benefit of acting as an immunomodulatory agent. There are published data indicating that this drug formulation sensitizes tumor cells for $\mathrm{T}$ cell-mediated, and possibly natural killer cell-mediated, apoptosis. ${ }^{40}$ Van der Most et al presented data demonstrating that immune sensitization from cyclophosphamide can augment treatment efficiency within combination therapy. ${ }^{40}$ Nevertheless, there are insufficient data on the proper time of initiation (ie, firstline, second-line).

MPM treatment has evolved during the past 20 years. In a previously published study, patients who received immediately initiated chemotherapy were associated with improved overall survival: $66 \%$ at 1 year compared to the "delayed" patients (36\%), whose quality of life was less well maintained. ${ }^{12}$ A new generation of chemotherapy drugs has been studied to improve survival benefits, but only pemetrexed in combination with cisplatin is an efficient first-line treatment, confirmed in phase III trials. This type of chemotherapy is the current standard therapy in advanced MPM. ${ }^{13}$ Multimodality treatment protocols have demonstrated a median survival of 19-46 months depending on the stage, histology, and completeness of the surgical resection. ${ }^{4-45}$ In regard to epithelial type, no lymph node involvement and complete surgical resection occasional long-term survival has been observed. ${ }^{41}$ Local disease control has been achieved with acceptable morbidity and mortality through extrapleural pneumonectomy and adjuvant high-dose hemithoracic irradiation. ${ }^{42,43,46}$

\section{Conclusion}

Despite the progression of clinical treatment, the data for MPM are not yet promising. The main clinical problem is probably the short duration of the response in chemotherapy and the early relapse of the disease due to chemoresistance. Most recent studies have focused on understanding the genetic events that contribute to the process of carcinogenesis from asbestos fibers and the molecular mechanisms responsible for the growth of cancer cells. Asbestos is responsible for the production of nitrogen species and active oxygen that can cause DNA damage and chromosome alterations. ${ }^{3,47}$ Its fibers may limit drug penetration by causing alterations of the stromal composition in neoplastic tissues. ${ }^{9}$ A better understanding of the molecular and immune pathways of carcinogenesis in MPM can lead to new targeted drugs and to a better medical treatment of the disease.

\section{Disclosure}

The authors report no conflicts of interest in this work. The authors acquired written informed consent from the patient to publish data and figures from his medical file.

\section{References}

1. Suemitsu R, Takeo S, Hamatake M, Furuya K, Uesugi N. A case of diffused malignant pleural mesothelioma forming small multiple disseminations with intraoperatively suspicious carcinoid tumors. Ann Thorac Cardiovasc Surg. 2011;17(3):290-292.

2. Rascoe PA, Jupiter D, Cao X, Littlejohn JE, Smythe WR. Molecular pathogenesis of malignant mesothelioma. Expert Rev Mol Med. 2012; 14:e12.

3. Matsuzaki H, Maeda M, Lee S, et al. Asbestos-induced cellular and molecular alteration of immunocompetent cells and their relationship with chronic inflammation and carcinogenesis. J Biomed Biotechnol. 2012;2012:492608. 
4. Pasello G, Altavilla G, Bonanno L, Rea F, Favaretto AG. A pathological complete response after preoperative chemotherapy with carboplatin and pemetrexed in malignant pleural mesothelioma: a case report. J Thorac Dis. 2010;2(4):254.

5. Pinton G, Manente AG, Angeli G, Mutti L, Moro L. Perifosine as a potential novel anti-cancer agent inhibits EGFR/MET-AKT axis in malignant pleural mesothelioma. PLoS One. 2012;7(5):e36856.

6. van der Bij S, Koffijberg H, Burgers JA, et al. Prognosis and prognostic factors of patients with mesothelioma: a population-based study. $\mathrm{Br} J$ Cancer. 2012;107(1):161-164.

7. Bagheri R, Haghi SZ, Rahim MB, Attaran D, Toosi MS. Malignant pleural mesothelioma: clinicopathologic and survival characteristic in a consecutive series of 40 patients. Ann Thorac Cardiovasc Surg. 2011; 17(2):130-136.

8. Betta PG, Magnani C, Bensi T, Trincheri NF, Orecchia S. Immunohistochemistry and molecular diagnostics of pleural malignant mesothelioma. Arch Pathol Lab Med. 2012;136(3):253-261.

9. Arrieta O, Medina LA, Estrada-Lobato E, et al. First-line chemotherapy with liposomal doxorubicin plus cisplatin for patients with advanced malignant pleural mesothelioma: phase II trial. Br J Cancer. 2012; 106(6):1027-1032.

10. Gabizon A, Shmeeda H, Grenader T. Pharmacological basis of pegylated liposomal doxorubicin: impact on cancer therapy. Eur J Pharm Sci. 2012;45(4):388-398.

11. Hillegass JM, Blumen SR, Cheng K, et al. Increased efficacy of doxorubicin delivered in multifunctional microparticles for mesothelioma therapy. Int J Cancer. 2011;129(1):233-244.

12. Sugarbaker DJ, Flores RM, Jaklitsch MT, et al. Resection margins, extrapleural nodal status, and cell type determine postoperative long-term survival in trimodality therapy of malignant pleural mesothelioma: results in 183 patients. J Thorac Cardiovasc Surg. 1999;117(1):54-63.

13. Vogelzang NJ. Standard therapy for the treatment of malignant pleural mesothelioma. Lung Cancer. 2005;50(Suppl 1):S23-S24.

14. Umsawasdi T, Dhingra HM, Charnsangavej C, Luna MA. A case report of malignant pleural mesothelioma with long-term disease control after chemotherapy. Cancer. 1991;67(1):48-54.

15. Zauderer MG, Krug LM. The evolution of multimodality therapy for malignant pleural mesothelioma. Curr Treat Options Oncol.2011;12(2) 163-172.

16. Alexopoulos EC. Occupational health services in Greek hospitals. Med Sci Monit. 2006;12(10):LE20-LE21.

17. Gogou E, Kerenidi T, Chamos V, Zintzaras E, Gourgoulianis KI. Mesothelioma mortality in Greece from 1983 to 2003. Int J Clin Pract 2009;63(6):944-948.

18. Northfelt DW, Dezube BJ, Thommes JA, et al. Efficacy of pegylatedliposomal doxorubicin in the treatment of AIDS-related Kaposi's sarcoma after failure of standard chemotherapy. J Clin Oncol. 1997; 15(2):653-659.

19. Uziely B, Jeffers S, Isacson R, et al. Liposomal doxorubicin: antitumor activity and unique toxicities during two complementary phase I studies. J Clin Oncol. 1995;13(7):1777-1785.

20. Sorensen PG, Bach F, Bork E, Hansen HH. Randomized trial of doxorubicin versus cyclophosphamide in diffuse malignant pleural mesothelioma. Cancer Treat Rep. 1985;69(12):1431-1432.

21. Baas P, van Meerbeeck J, Groen H, et al. Caelyx in malignant mesothelioma: a phase II EORTC study. Ann Oncol. 2000;11(6):697-700.

22. Allen TM, Chonn A. Large unilamellar liposomes with low uptake into the reticuloendothelial system. FEBS Lett. 1987;223(1):42-46.

23. Gabizon A, Papahadjopoulos D. Liposome formulations with prolonged circulation time in blood and enhanced uptake by tumors. Proc Natl Acad Sci U SA. 1988;85(18):6949-6953.

24. Papahadjopoulos D, Gabizon A. Targeting of liposomes to tumor cells in vivo. Ann N Y Acad Sci. 1987;507:64-74.

25. Huang SK, Martin FJ, Jay G, Vogel J, Papahadjopoulos D, Friend DS. Extravasation and transcytosis of liposomes in Kaposi's sarcoma-like dermal lesions of transgenic mice bearing the HIV tat gene. Am J Pathol. 1993;143(1):10-14.
26. Zarogoulidis K, Kontakiotis T, Hatziapostolou P, et al. A Phase II study of docetaxel and carboplatin in the treatment of non-small cell lung cancer. Lung Cancer. 2001;32(3):281-287.

27. de Cupis A, Semino C, Pirani P, Loprevite M, Ardizzoni A, Favoni RE. Enhanced effectiveness of last generation antiblastic compounds vs cisplatin on malignant pleural mesothelioma cell lines. Eur $J$ Pharmacol. 2003;473(2-3):83-95.

28. Huang Z, Byun B, Matsubara T, Moskowitz MA. Time-dependent blockade of neurogenic plasma extravasation in dura mater by 5-HT1B/D agonists and endopeptidase 24.11. Br J Pharmacol. 1993; 108(2):331-335.

29. Chen GL, Yang L, Rowe TC, Halligan BD, Tewey KM, Liu LF. Nonintercalative antitumor drugs interfere with the breakagereunion reaction of mammalian DNA topoisomerase II. J Biol Chem. 1984;259(21):13560-13566.

30. O'Dwyer PJ, Leyland-Jones B, Alonso MT, Marsoni S, Wittes RE Etoposide (VP-16-213). Current status of an active anticancer drug. N Engl J Med. 1985;312(11):692-700.

31. Clark PI, Slevin ML. The clinical pharmacology of etoposide and teniposide. Clin Pharmacokinet. 1987;12(4):223-252.

32. Slevin ML, Clark PI, Joel SP, et al. A randomized trial to evaluate the effect of schedule on the activity of etoposide in small-cell lung cancer. J Clin Oncol. 1989;7(9):1333-1340.

33. Clark PI, Slevin ML, Joel SP, et al. A randomized trial of two etoposide schedules in small-cell lung cancer: the influence of pharmacokinetics on efficacy and toxicity. J Clin Oncol. 1994;12(7):1427-1435.

34. Zarogoulidis K, Mylonaki E, Kakavelas P, et al. Topotecan-carboplatinetoposide combination as 1st line treatment in patients with small cell lung cancer. Lung Cancer. 2009;66(2):226-230.

35. Sahmoud T, Postmus PE, van Pottelsberghe C, et al. Etoposide in malignant pleural mesothelioma: two phase II trials of the EORTC Lung Cancer Cooperative Group. Eur J Cancer. 1997;33(13):2211-2215.

36. Planting AS, van der Burg ME, Goey SH, et al. Phase II study of a short course of weekly high-dose cisplatin combined with long-term oral etoposide in pleural mesothelioma. Ann Oncol. 1995;6(6):613-615.

37. Tammilehto L, Maasilta P, Mantyla M, Salo J, Mattson K. Oral etoposide in the treatment of malignant mesothelioma. A phase II study. Ann Oncol. 1994;5(10):949-950.

38. Anderson H, Hasleton P, Michie AB, Johnson RJ, Thatcher N. 24-hour cyclophosphamide infusion therapy for malignant mesothelioma of the pleura. Br J Dis Chest. 1988;82(1):64-69.

39. Shin DM, Fossella FV, Umsawasdi T, et al. Prospective study of combination chemotherapy with cyclophosphamide, doxorubicin, and cisplatin for unresectable or metastatic malignant pleural mesothelioma. Cancer. 1995;76(11):2230-2236.

40. van der Most RG, Currie AJ, Cleaver AL, et al. Cyclophosphamide chemotherapy sensitizes tumor cells to TRAIL-dependent CD8 $\mathrm{T}$ cell-mediated immune attack resulting in suppression of tumor growth. PLoS One. 2009;4(9):e6982.

41. Rusch VW, Rosenzweig K, Venkatraman E, et al. A phase II trial of surgical resection and adjuvant high-dose hemithoracic radiation for malignant pleural mesothelioma. J Thorac Cardiovasc Surg. 2001; 122(4):788-795.

42. Rea F, Marulli G, Bortolotti L, et al. Induction chemotherapy, extrapleural pneumonectomy (EPP) and adjuvant hemi-thoracic radiation in malignant pleural mesothelioma (MPM): feasibility and results. Lung Cancer. 2007;57(1):89-95.

43. Pagan V, Ceron L, Paccagnella A, Pizzi G. 5-year prospective results of trimodality treatment for malignant pleural mesothelioma. J Cardiovasc Surg (Torino). 2006;47(5):595-601.

44. Flores RM, Zakowski M, Venkatraman E, et al. Prognostic factors in the treatment of malignant pleural mesothelioma at a large tertiary referral center. J Thorac Oncol. 2007;2(10):957-965.

45. Flores RM, Krug LM, Rosenzweig KE, et al. Induction chemotherapy, extrapleural pneumonectomy, and postoperative high-dose radiotherapy for locally advanced malignant pleural mesothelioma: a phase II trial J Thorac Oncol. 2006;1(4):289-295. 
46. Ahamad A, Stevens CW, Smythe WR, et al. Promising early local control of malignant pleural mesothelioma following postoperative intensity modulated radiotherapy (IMRT) to the chest. Cancer J. 2003; $9(6): 476-484$.
47. Carbone M, Ly BH, Dodson RF, et al. Malignant mesothelioma: facts, myths, and hypotheses. J Cell Physiol. 2012;227(1):44-58.

\section{Publish your work in this journal}

OncoTargets and Therapy is an international, peer-reviewed, open access journal focusing on the pathological basis of all cancers, potential targets for therapy and treatment protocols employed to improve the management of cancer patients. The journal also focuses on the impact of management programs and new therapeutic agents and protocols on patient perspectives such as quality of life, adherence and satisfaction. The manuscript management system is completely online and includes a very quick and fair peer-review system, which is all easy to use. Visit http://www.dovepress.com/testimonials.php to read real quotes from published authors.

\footnotetext{
Submit your manuscript here: http://www.dovepress.com/oncotargets-and-therapy-journal
} 